\title{
pÿCharacterization of membrane foulant interactions with novel combination of Raman spectroscopy, surface plasmon resonance and molecular dynamics simulation
}

\section{Virtanen, Tiina}

2018-10-31

Virtanen , T , Parkkila , P , Koivuniemi , A, Lahti , J , Viitala , T , Kallioinen , M , Mänttäri , M pÿ\& Bunker , A 2018 , ' Characterization of membrane foulant interactions with novel combination of Raman spectroscopy, surface plasmon resonance and molecular dynamics simulation ', Separation and Purification Technology , vol. 205 , pp. 263-272 . https://doi.org/10.1016/j.seppur.2018.

http://hdl.handle.net/10138/325076

https://doi.org/10.1016/j.seppur.2018.05.050

cc_by_nc_nd

acceptedVersion

Downloaded from Helda, University of Helsinki institutional repository.

This is an electronic reprint of the original article.

This reprint may differ from the original in pagination and typographic detail.

Please cite the original version. 


\title{
Characterization of membrane-foulant interactions with novel combination of Raman spectroscopy, surface plasmon resonance and molecular dynamics simulation
}

\author{
Tiina Virtanen ${ }^{\mathrm{a}}$, Petteri Parkkila ${ }^{\mathrm{b}}$, Artturi Koivuniemi $^{\mathrm{b}}$, Jussi Lahti ${ }^{\mathrm{a}}$, \\ Tapani Viitala ${ }^{\mathrm{b}}$, Mari Kallioinen ${ }^{\mathrm{a}}$, Mika Mänttäri ${ }^{\mathrm{a}}$, Alex Bunker ${ }^{\mathrm{b}, *}$ \\ ${ }^{a}$ Laboratory of Membrane Technology and Technical Polymer Chemistry, LUT School of \\ Engineering Science, Lappeenranta University of Technology, Lappeenranta, Finland \\ ${ }^{b}$ Division of Pharmaceutical Biosciences, Faculty of Pharmacy, University of Helsinki, \\ Helsinki, Finland
}

\begin{abstract}
Adsorptive fouling, by phenolic compounds, is a serious issue regarding the development and use of membrane based filtration technologies for water purification and wastewater treatment. We have developed a novel, combined, protocol of Raman spectroscopy and surface plasmon resonance (SPR) experiments, along with molecular dynamics (MD) simulation, to study the interaction of vanillin, a model phenolic compound, with the polyethersulfone (PES) surface of a membrane. The adsorption of vanillin to the PES surface was found to be highly $\mathrm{pH}$ dependent; the source of this was determined, by MD simulation, to be the stronger interaction with the protonated form of vanillin, predominant at low $\mathrm{pH}$. Vanillin interacts with the PES surface, both through entropy driven, hydrophobic, interactions and, for the case of the protonated form, H-bonding of the hydroxyl group with the sulphur oxygens of the PES molecules. In addition to general insight into the fouling process that can be used to develop new methods to inhibit adsorptive fouling, our results also elucidate the specific interaction of the PES membrane with vanillin that can be used in the development of anti-fouling coatings, based on the structure of vanillin.
\end{abstract}

Keywords: fouling, adsorption, label-free analytics, polymer membrane,

\footnotetext{
${ }^{*}$ Corresponding author

Email address: alex.bunker@helsinki.fi (Alex Bunker )
} 
spin coating

\section{Introduction}

Fouling, the adhesion of foreign materials to a surface in a fluid medium, has emerged as a serious problem regarding the development and application of membrane based filtration technologies [1-7]. Due to fouling, membrane performance can be severely impeded through both 1) a reduction of the pore size, changing the filtration behavior [1] and 2) altering the surface properties of the membrane, e.g. hydrophobicity and surface charge [2].

Membranes generally have a selective surface, skin layer, composed of relatively rigid polymers; polyethersulfone (PES) or polyvinylidene fluoride (PVDF) are common choices, possibly with trace quantities of additives, to adjust the properties of the surface [8], e.g. permanently hydrophilic polyethersulfone (PESH). These materials, capable of forming ion-dipole interactions or H-bonding with water [9], are also, however, generally relatively hydrophobic [10]. As a result of their specific properties, surfaces composed of these polymers are very susceptible to fouling.

Adsorptive fouling is challenging to control. This process is governed by weak intermolecular forces, i.e. non-bonded interactions, between the foulant molecules and the polymers that form the skin layer of the membrane $[11,12]$. Clearly, the concentration of foulant molecules in the fluid medium and the nature of their direct interaction with the surface, both play a role; several properties of the fluid environment, in which the membrane is immersed, however, also govern the extent to which absorptive fouling occurs. These include, hydrodynamics, i.e. flow conditions, $\mathrm{pH}$ and the presence of other molecules that either accelerate or inhibit the fouling process [13]. A detailed mechanistic understanding of these factors is critical to the development of methods to improve membrane performance through the control of this phenomenon.

Abbreviations: DFT $=$ density functional theory, $K_{D}=$ dissociation constant, $\mathrm{MD}=$ molecular dynamics, $\mathrm{SPR}=$ surface plasmon resonance, $\mathrm{TIR}=$ total internal reflectance, $\mathrm{PES}=$ polyethersulfone, $\mathrm{PESH}=$ permanently hydrophilic polyethersulphone, $\mathrm{ATR}=$ attenuated total reflectance, $\mathrm{PVDF}=$ polyvinylidene fluoride, $\mathrm{PCA}=$ principal component analysis 
A specific class of molecules that both PES and PVDF skin layers are vulnerable to adsorptive fouling from, are phenolic and ligneous compounds [1416]; they are commonly present in the fluid environments in which these membranes operate. Phenolic compounds, like humic acid, [17] are abundant in surface water, thus an important source of fouling in relation to the use of membrane based technologies for drinking water purification [17]; lignin related compounds are common in many wastewater streams, [18] e.g., effluent from pulp bleaching [19, 20], where filtration using membranes has been proposed [21]. Overall, separation and removal of these specific compounds is of significant industrial importance [11, 22]. For example, Koivula et al. [15] found the filtration of birch hydrolysate, using a commercial ultrafiltration membrane, to be severely impeded due to the occurrence of acute and rapid fouling; the resultant flux at 5.5 bar and $60{ }^{\circ} \mathrm{C}$ was reduced to only $2 \mathrm{Lm}^{-2} \mathrm{~h}^{-1}$. Birch hydrolysate is known to contain phenolic ligneous compounds; the filtration efficiency was found to be significantly enhanced when these were removed: the ligneous compounds were shown to play the key role in the fouling that was observed. Other groups have also found evidence of adsorptive fouling of ultrafiltration membranes by phenolic compounds $[22,23]$.

In possession of both polar and non-polar structural units, phenolic compounds are amphipathic; the hallmark of their structure is the possession of both hydrophobic phenyl rings and hydrophilic OH-groups; these can act as H-bond donors at low $\mathrm{pH}$, in their protonated state and, at higher $\mathrm{pH}$, become ionized (deprotonated), thus more hydrophilic. The mechanism through which they adsorb to the polymer film is thus a combination of both electrostatic, e.g. H-bonding and entropy driven, i.e. hydrophobic, effects $[9,24]$. Normally, these forms of interaction counteract one another, however, in this case they both work together to increase binding; this is the source of their potency as agents of adsorptive fouling. Susanto et al. [22] studied these interactions in detail and proposed that adsorption is an extremely complex process, involving many different interaction mechanisms, including $\mathrm{H}$-bonding, hydrophobic interactions, $\pi-\pi$ stacking of benzene rings and water ordering at the membrane surface. The methodology used by this group did not, however, enable definitive confirmation of what they proposed.

In this study, a novel combination of two experimental methodologies: Raman spectroscopy and surface plasmon resonance (SPR), with computational molecular dynamics modelling (MD) has been applied to obtain further insight into the mechanisms that govern the adsorptive fouling of PES 
based filtration membranes by phenolic compounds; the goal is to provide insight that could lead to the development of new methods to inhibit this phenomenon. Specifically, the effect of $\mathrm{pH}$ and the mechanisms involved in this process have been studied. Vanillin was selected as a model phenolic compound that, additionally, has itself been proposed as an antifouling agent [25-28]. Vanillin, a degradation product of wood lignin, is a weak acid with a hydroxyl group that is protonated at acidic $\mathrm{pH}$ and unprotonated at basic $\mathrm{pH}$.

Using Raman spectroscopy in real time, the interaction of vanillin with a commercial ultrafiltration membrane was investigated: the UH004 P (MicrodynNadir GmBH) membrane, with a skin layer composed of PESH. The interaction of vanillin with a pure PES surface was also examined, using SPR, not involving a cross-filtration system as in Raman spectroscopy experiments. PESH only differs from PES in the addition of a small quantity of hydrophilic polymer, to control pore structure and increase the hydrophobicity of the surface. Thus, a PES surface can be seen as an appropriate model of the PESH surface of a commercial ultrafiltration membrane. The structure of both PES and vanillin are shown in Fig. 1.

The protonation state of the vanillin molecule, thus $\mathrm{pH}$, clearly plays a role in the nature of the interactions between vanillin and the PES surface. Unprotonated vanillin is more hydrophilic than the protonated form, thus it would be expected to have a decreased tendency to adhere to the PES film. Specific interactions between different functional groups of vanillin and PES are also expected to play a role. It can plausibly be postulated that protonated vanillin molecules possess the capacity to form $\mathrm{H}$-bonds with either the ether or sulphur oxygens of PES. This mechanism could further enhance the strength of the interaction between PES and vanillin in a fashion typical for phenolic compounds. As mentioned above, both electrostatic and entropic effects would be involved in its interaction with the membrane surface. The specific research questions addressed by this study are shown in schematic form in Fig. 1.

Raman spectroscopy is a minimally invasive spectroscopic technique that has been widely used for off-line identification and characterization of chemical structures and to determine, quantitatively, the amount of analyte in a sample; it has also been used in a wide range of applications relating to process and synthesis monitoring [29]. It is possible to identify a wide range of organic compounds and functional groups from their absorption spectra; the intensity of each spectra can be used to determine the quantity of each 


\section{Vanillin molecule}<smiles>COc1cc(C=O)ccc1O</smiles>

Protonated vs. Unprotonated

Aggregation at PES surface?
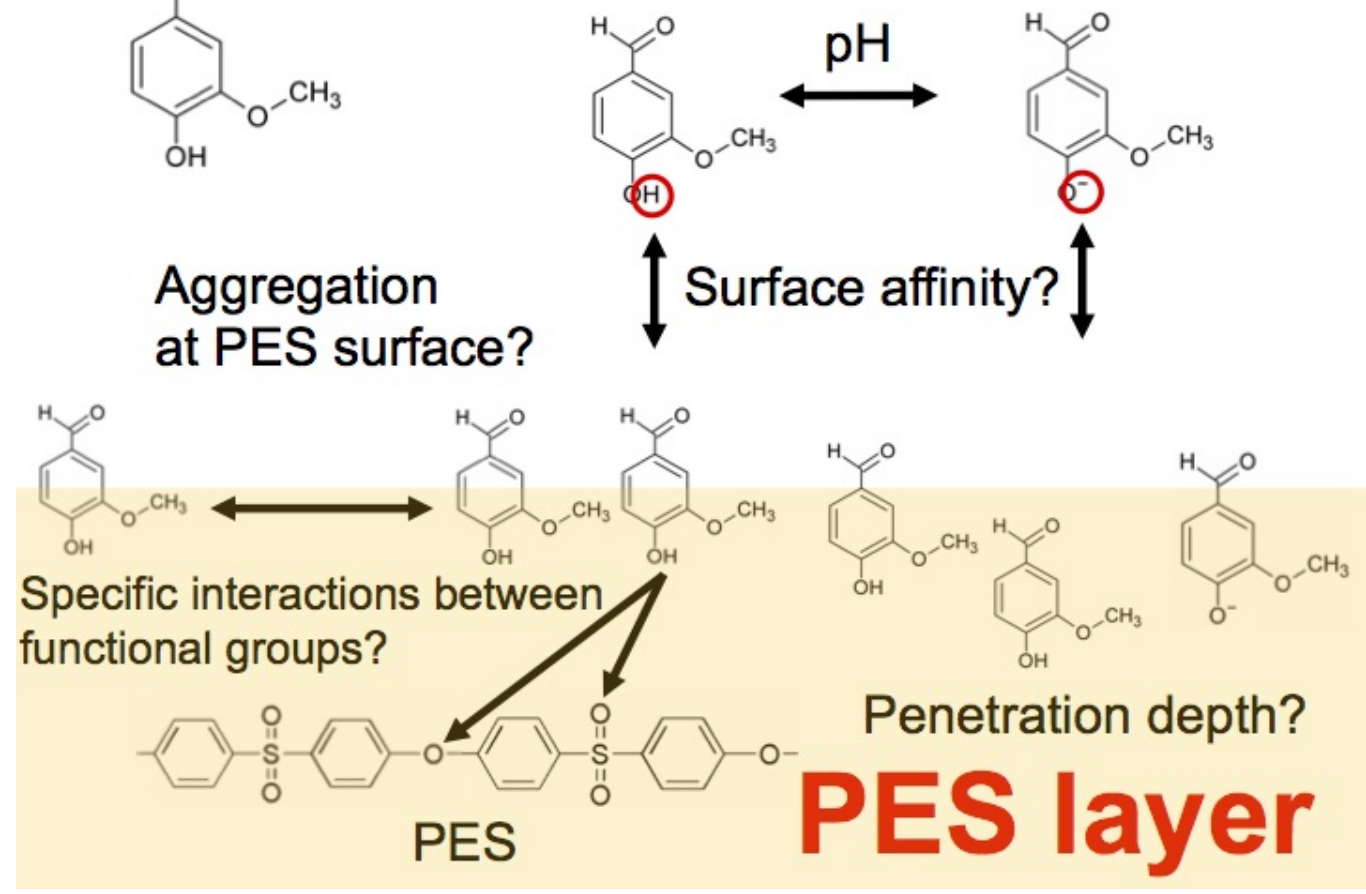

Figure 1: Schematic of the system studied and the research questions asked, concerning the interaction of vanillin molecules with a PES film.

compound adsorbed to a given surface [30]. In previous published work [31], we have shown that vanillin adsorbs to PESH membranes and the quantity absorbed can be monitored in real time using Raman spectroscopy.

Analogous to the intensity of a Raman spectra, the strength of the response signal from an SPR experiment can be directly correlated to the extent of a deposited layer on a surface [32-34]. However, it should be noted that the SPR protocol cannot be used to directly study the surface of a commercial ultrafiltration membrane, as the technique requires spin coating the polymer that comprises the model surface directly onto a sensor chip. Thus, SPR serves only as a protocol to study adsorption and desorption of compounds to the spin coated model surface and the properties of the bulk solution above the interface. Further details regarding the SPR protocol are found in the Supplementary Material (SM), see Fig. S1. Both Raman spectroscopy and SPR are label free; they do not involve the insertion of any 
probe molecule into the system. As such, they are, while extremely sensitive to even the smallest changes in surface concentration, able to investigate a surface without the corrupting effect of the introduction of a new molecule.

Molecular dynamics simulation (MD) can provide a three dimensional picture of the system being studied; a section of the PES surface, $\sim 15 \times 15 \mathrm{~nm}$ in size, can be observed over a timescale of hundreds of nanoseconds. As the result is only a model, the stand alone value is, admittedly, limited, however, when combined with the aforementioned experimental analysis, a window onto the system is opened that adds mechanistic insight to the experimental results; it can be seen, as described in a review written by Lee et al. [35], as a "computational microscope" focussed on the system being studied. In this study, a synergistic combination of all three of these methodologies, Raman spectroscopy, SPR and MD simulation, has been used to study this system.

\section{Materials and methods}

\subsection{Vanillin: the model compound}

Vanillin is a moderately lipophilic molecule with an octanol-water partition coefficient of $1.37(\log \mathrm{P})$, thus has greater affinity for hydrophobic, rather than hydrophilic, environments. This $\log \mathrm{P}$ value, however, describes the partitioning of the unionized, protonated, form of the molecule; vanillin is in possession of a hydroxyl group with a pKa of 7.4 , thus it is a weak acid: the percentages of protonated vanillin will be $100 \%, 50 \%$ and $0.25 \%$ at pH values of 2, 7.4 and 10, respectively; both the Raman spectroscopy and SPR experiments were carried out at these three $\mathrm{pH}$ values.

\subsection{Raman monitoring of the adsorption of vanillin on the surface of the UH004 P PESH membrane}

\subsubsection{Materials}

The UH004 P membrane (Microdyn-Nadir GmBH) that has a skin layer composed of PESH and a molecular weight cut-off of $4 \mathrm{kDa}$ was used in adsorption studies. Vanillin (4-hydroxy-3-methoxybenzaldehyde, 99 \%) was purchased from Acros Organics. pH was adjusted using sodium hydroxide (Merck, $\geq 99.0 \%$ ) and hydrochloric acid (VWR, 37 \%, European Pharmacopoeia quality standard) solutions. Methanol (J. T. Baker, min. $99.8 \%$ ) was used as an extraction solvent. Pure water $(15 \mathrm{M} \Omega \mathrm{cm})$ was obtained from CENTRA-R 60/120 system (Elga Veolia Water Solutions). 


\subsubsection{Cross-flow experiments}

The experiments were carried out using cross-flow flat-sheet modules. A schematic diagram of the experimental protocol is shown in Fig. 2; the protocol involved two such modules, labeled (A) and (B) in the figure; module (A) was always flushed after the adsorption was completed, while module (B) was not. The effective membrane surface area was $45 \mathrm{~cm}^{2}$. A heat exchanger was used to maintain a temperature of $25 \pm 0.3{ }^{\circ} \mathrm{C}$. The adsorption step was carried out using a peristaltic pump $(0.4 \mathrm{~m} / \mathrm{s})$. Cross-flow velocity was adjusted to $1.5 \mathrm{~m} / \mathrm{s}$ during pressurizing, pure water permeability measurements and, for the case of module (A), flushing after the adsorption.

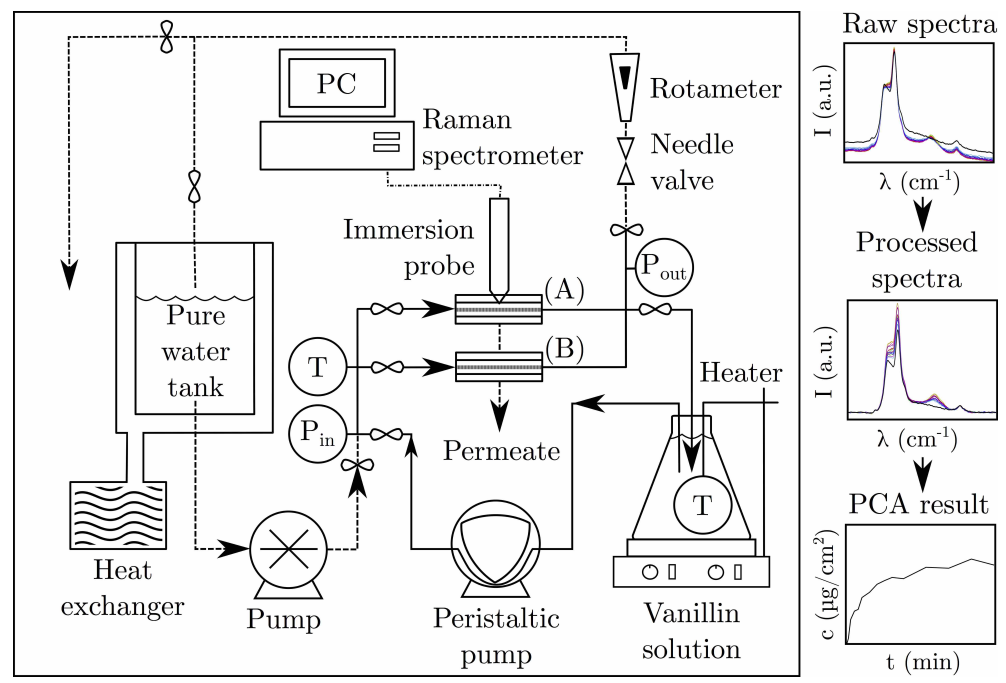

Figure 2: Schematic of the cross-flow filtration system combined with the Raman sampling setup.

In all cases, the membranes were initially treated by filtering pure water, under a pressure of $3 \mathrm{bar}$, to remove all preservatives. In order to compact the membrane, the transmembrane pressure was then increased to 5 bar for a period of 30 minutes. The pure water flux was then measured at a pressure of 3 bar, until the flux stabilized. Following this, the adsorption experiments were performed without applied pressure, at a vanillin concentration of $0.75 \mathrm{~g} / \mathrm{L}$; in our previous study [31], we found this concentration to be sufficient to obtain a quantitative result from both methods of spectroscopic analysis. The solutions were circulated for 120 minutes; the experiment was repeated three times at $\mathrm{pH} 2$ and 7.4 and twice at $\mathrm{pH} 10$. 
Following the adsorption phase of the experiment, the membranes in the Raman monitoring module (A) were flushed with pure water, at a pressure of 3 bar, until the conductivity had been observed to decrease below $3 \mu \mathrm{S} / \mathrm{cm}$, then stabilized for 15 minutes; following this, pure water permeability measurements were repeated. All membranes, both the flushed, (A) and not flushed, (B), were finally dried at room temperature and subjected for ATRFTIR analysis and for external calibration through extraction and UV/Vis analysis.

\subsubsection{Raman monitoring and data processing}

Real-time Raman monitoring was performed, using a Kaiser RXN1 Raman spectrometer at $785 \mathrm{~nm}$ laser excitation with a maximum power of 400 $\mathrm{mW}$ [31]. Raman immersion MR probe with focal distance of $0 \mathrm{~mm}$ was inserted above the surface of the membrane. Raman spectra were obtained as three, separate, three second long accumulations. Spectral data was processed using MATLAB (v. R2016a). Baseline fits were determined, using linear interpolation and subtracted from the original spectra. The resulting spectra were normalized with respect to the membrane peak at $1071.6 \mathrm{~cm}^{-1}$.

The preprocessed Raman spectra were analyzed using the most simple multivariate chemometrical method, principal component analysis (PCA) [31] in order to obtain concentration profiles for the adsorption of vanillin, as a function of time. The PCA "score" provides a visualization of the evolution of the amount of adsorbed substrate, at each time point where a measurement is taken. PCA analysis was performed separately for each adsorption experiment. The first two principal components were used for our analysis. Since PCA was applied to an already normalized spectra, no additional scaling was performed. The external calibration method [31], i.e. extraction and UV/Vis analysis, was used for the scaling of the PCA score results to obtain a quantitative result for the adsorbed vanillin surface density, i.e. mass per surface area, as a function of time. For scaling the concentration of vanillin in the beginning of the adsorption experiment was set to zero (0 $\left.\mu \mathrm{g} / \mathrm{cm}^{2}\right)$. The concentration following adsorption was taken from extraction results and simple regression calculation was used to scale the score values as explained in detail in the previous study [31].

\subsubsection{Infrared spectroscopic analysis}

Infrared (IR) and Raman spectroscopy are complementary techniques for the study of molecular vibrations and structures [30]. Thus, Attenuated Total 
Reflectance Fourier Transform Infrared Spectroscopy (ATR-FTIR) was carried out as a supplementary analysis methodology, using the samples present at the end of the experiment, once the system had been dried and, in some cases, also flushed; this allowed for us to obtain a separate measure of the total quantity of vanillin adsorbed to the PESH membrane.

Both flushed (module A) and unflushed (module B) membranes were analyzed, using Perkin-Elmer Frontier spectrometer, to characterize the changes caused by vanillin adsorption, in the range of $400-4000 \mathrm{~cm}^{-1}$. The FTIR analysis was carried out in the ATR mode, equipped with a diamond crystal. The resolution was $4 \mathrm{~cm}^{-1}$ and for each spectrum 20 scans were collected, with a data interval of $1 \mathrm{~cm}^{-1}$. The scans were co-added, ATR corrected, baseline corrected and normalized. For each membrane, 10 spectra were measured, each measurement taken on a different point on the membrane surface.

\subsubsection{Extraction of vanillin from membrane and $U V / V i s$ analysis}

The quantity of adsorbed vanillin on the membranes was assessed through extraction and UV/Vis analysis as described in the previous study [31]. Three membrane samples, each with an area of $6.6 \mathrm{~cm}^{2}$, were cut from each membrane. All membrane samples were extracted until all vanillin desorbed, either three or four times, using $3 \mathrm{~mL}$ aliquots of methanol (Merck, $\geq 99.5 \%$ ) and the assistance of an ultrasonic bath (USF Finnsonic W 181).

The extracts of each sample were combined and the absorption spectra of the combined extracts were recorded, using a Jasco V-670 spectrophotometer, at room temperature. Quartz cells, with $1 \mathrm{~cm}$ path lengths, were used. The absorption wavelength for vanillin (308 nm) was used for excitation; five accumulations were collected with a bandwidth of $2.0 \mathrm{~nm}$. The molar absorptivity of vanillin in methanol solution, $\varepsilon=8912 \mathrm{~cm}^{-1} \mathrm{M}^{-1}$ [36], was used for concentration calculations.

\subsection{SPR analysis of adsorption of vanillin to a model PES surface}

\subsubsection{Materials}

Polyethersulfone granules (SU306311, Goodfellow Cambridge Ltd, Huntingdon, UK) with molecular weight of $58000 \mathrm{~g} / \mathrm{mol}$ and vanillin (4-hydroxy3-methoxybenzaldehyde, 99 \%) were purchased from Sigma-Aldrich. Dichloromethane was obtained from Fluka/Sigma-Aldrich. Deionized water (resistivity of 18.2 M $\Omega \mathrm{cm}$ ), from a Milli-Q water purification system (Merck KGaA, Darmstadt, Germany), was used in all cases for sample and running buffer prepara- 
tion. Polyethersulfone granules were dissolved in dichloromethane $(0.5 \% \mathrm{w} / \mathrm{v})$ and the solution was kept overnight, at room temperature, to ensure complete dissolution. Vanillin stock solutions $(1 \mathrm{mg} / \mathrm{mL})$ were first prepared in deionized water, then samples were diluted to the desired concentration and, finally, $\mathrm{pH}(2,7.4$ or 10) was adjusted for each sample using $0.1 \mathrm{M}$ sodium hydroxide and hydrochloric acid solutions (Reagena, Toivala, Finland).

\subsubsection{Sensor coating}

Initial cleansing of the gold sensor slides was achieved, through immersion, in a boiling solution of hydrogen peroxide : ammonia : water (1:1:5 volume ratio) for five minutes. Between each measurement, the slides were wiped, with a dichloromethane-soaked tissue, then rinsed with ethanol and Milli-Q water, in order to remove any residual PES coating that remained from the previous measurement. Following this, $65 \mu \mathrm{L}$ of PES solution was adsorbed onto the sensor surface using spin coating [37]. This was performed for one minute with an angular velocity of $6300 \mathrm{rpm}$. Atomic force microscopy images and the polarization modulation-infrared reflection-adsorption spectrum of the PES coated surface, are available in SM, Fig. S2. The reproducibility of our coating procedure was established through the application of fifteen independent depositions onto separate sensors, in each case the SPR peak angular position was measured, before and after deposition of PES onto the SPR sensor chip.

\subsubsection{SPR measurements}

Measurements were made using an automated, multi parametric (MP), MP-SPR Navi ${ }^{\text {TM }} 220$ A (BioNavis Ltd., Tampere, Finland) instrument, equipped with two independent flow channels and two laser wavelengths $(670 \mathrm{~nm}$ and $785 \mathrm{~nm}$ ). Detailed schematic of the SPR protocol can be found in the SM, Fig. S1. The sensor surface, coated with PES, was inserted into a flow cell containing a solution of vanillin. This system was monitored using an incident laser beam, as the vanillin adsorbed from the bulk solution to the PES surface. The quantitative signal that defines the SPR response in our measurements is known as the SPR peak angular shift (see Fig. S1, SM), measured in millidegrees. The SPR peak angle, obtained before the vanillin solution was added to the flow medium, was used as the baseline for the SPR response to vanillin adsorption. While the traditional SPR devices are only capable of measuring reflectivity of light at a fixed angle, MP-SPR is capable of monitoring the entire range of incident angles (typically 50-78 ) 
simultaneously. As a result, in addition to the aforementioned SPR peak angular shift, the angle of total internal reflection (TIR, shown in Fig. S1, $\mathrm{SM}$ ), that provides information regarding the optical properties of the bulk solution, is also an output of MP-SPR.

The interaction kinetics of vanillin, at six separate concentration levels $(25,75,225,450,675$ and $1000 \mu \mathrm{g} / \mathrm{mL}$ and three $\mathrm{pH}$ values $(2,7.4$ and 10), was measured. The bulk liquid had the same $\mathrm{pH}$ values as the vanillin samples. Vanillin solutions were allowed to interact with the PES coating for 10 minutes, after which running buffer was injected into the flow channels, in order to monitor the dissociation of vanillin from the surface. For each sample, three independent measurements were performed and the average SPR responses, along with their respective standard deviations, were calculated. All measurements were performed at a constant temperature of $20{ }^{\circ} \mathrm{C}$; the flow rate was maintained at $40 \mu \mathrm{L} / \mathrm{min}$. We observed no apparent difference in the adsorption kinetics with either the lower $(20 \mu \mathrm{L} / \mathrm{min})$ or higher $(50 \mu \mathrm{L} / \mathrm{min})$ flow rates during test measurements; this excludes the possibility of mass transfer and diffusion-related effects near the sample surface.

\subsubsection{Data analysis}

When we analyzed the binding kinetics, our results showed large deviations from the standard one-to-one (1:1) model, corresponding to a single dissociation constant. We thus utilized a more complex one-to-two (1:2) binding model that assumes the scenario of two separate and independent binding mechanisms; this approach proved fruitful: binding between PES and vanillin thus involves two separate mechanisms with different affinities, one stronger than the other, thus two dissociation constants $K_{D 1}$ and $K_{D 2}$. The significance of this result is discussed later. In this model, the differential equations, describing the one-to-one system, are still valid, however, the contributions from both binding mechanisms are summed [38].

The dissociation constants, at all measured concentrations, for both binding mechanisms, $K_{D 1}$ (strong binding) and $K_{D 2}$ (weak binding), were obtained through numerically fitting the corresponding differential equations to the SPR data sets, including both the association and dissociation curves; in principle, the dissociation constants represent the concentrations at which half of the corresponding binding sites are occupied. TraceDrawer software, version 1.7 (RidgeView Instruments Ltd, Uppsala, Sweden) was used for the calculation of 1:2 kinetic model parameters and other analysis was per- 
formed using MATLAB (v. R2016a, The MathWorks, Natick Massachusetts, USA) and Origin Pro (v. 8.6.0, Origin Lab Corporation, Northampton Massachusetts, USA) software packages.

\section{4. $M D$ simulation}

\subsubsection{Summary of MD simulations performed}

Computational MD simulation provides us with the means to directly investigate the interaction of both protonated and unprotonated forms of vanillin, with the PES surface, to obtain mechanistic insight into the $\mathrm{pH}$ dependence of the affinity of vanillin for the $\operatorname{PES}(\mathrm{H})$ membrane. We thus simulated both forms of vanillin molecules at the interface of water and a matrix of PES dimers. While, for the experimental system, PES is a very long polymer, we simulated a matrix of PES dimers, as a tractable model, to study the microscopic interactions between PES and vanillin. Two separate systems were simulated 1) a layer of PES interacting with 20 vanillin molecules, 10 protonated and 10 not protonated that we refer to as system "VAN20" and 2) a layer of PES interacting with 50 protonated vanillin molecules, known as "VAN50". For the case of the VAN20 system, charge neutrality was achieved through adding sodium ions to the simulation; sodium ions were chosen, since $\mathrm{NaCl}$ is the most common salt to occur in the fluid environments in which filtration membranes operate.

\subsubsection{Forcefield development}

Interaction parameters, for both PES dimer and vanillin molecules, were developed using the OPLS all atom (OPLS-AA) force field [39]; the OPLSAA parameter set was used for all other molecules present in the simulation. This includes the TIP3P model for water [40]. The sodium ions were modelled using the OPLS-AA force field [39]. Partial charges for both PES and vanillin were generated using the Gaussian program (Revision E.01) [41] and the antechamber utility, included in the AMBER simulation package [42]. Models of both protonated and unprotonated forms of vanillin were built. Geometry optimization, of both PES and vanillin, was carried out, at the density functional theory level (DFT), employing the B3LYP hybrid functional, with the $6-31^{*}$ basis set. Tight convergence criteria was set during geometry optimization. After optimization, all electrostatic potentials were derived according to the Merz-Kollman scheme [43], with the same functional and basis set as used in geometry optimization. The antechamber program and the RESP (Restrained ElectroStatic Potential) fitting scheme 
were utilized to derive atomic partial charges that produce the electrostatic potential around the molecules, derived from the quantum mechanical calculations $[42,44]$. To derive Lennard-Jones and bonded force field parameters, we used the MKTOP script (v. 2.2.1) [45], a tool for creating AMBER03 and OPLS-AA molecular topologies, based on the covalent bonding network and functional groups of a molecule.

\subsubsection{System construction, simulation parameters and analysis}

In order to construct a model of a PES layer immersed in water, we initially constructed a system of 100 PES dimers in a box of dimensions $5 \times 5 \times 5 \mathrm{~nm}$. To obtain the initial configuration, first 5000 steps of energy minimization were carried out, using the steepest descent method, followed by $10 \mathrm{~ns}$ of simulation. Water was then added to the system (PES-W) through expansion of the simulation box, along the Z-axis, then filling the new volume; as a result the total number of water molecules was 1655 and the size of the periodic simulation box was $4 \times 4 \times 7.5 \mathrm{~nm}$. The resultant system was simulated for $300 \mathrm{~ns}$, to achieve an equilibrium conformation, at the end of which the surface tension was measured and found to be in approximate agreement with the experimental value for this property.

Two simulations were then performed with 20 (VAN20) and 50 (VAN50) vanillin molecules added to the water phase, respectively. For the case of VAN20, half of the vanillin molecules were unprotonated; their total charge was -1 . We added $10 \mathrm{Na}^{+}$counter ions to the VAN20 system to neutralize it. The number and type, of all molecules, in all systems, is found in Tab. 1. In addition, the number of water molecules in the VAN50 system was increased to 2708 , to solvate the larger number of vanillin molecules completely.

Table 1: molecular composition and simulation time for each simulated system

\begin{tabular}{lllll}
\hline System & PES & Water & Vanillin (prot./unprot.) & Time (ns) \\
\hline pure PES & 100 & 0 & 0 & 10 \\
\hline PES - Water & 100 & 1655 & 0 & 200 \\
\hline VAN20 & 100 & 1655 & $10 / 10$ & 320 \\
\hline VAN50 & 100 & 2708 & $50 / 0$ & 320 \\
\hline
\end{tabular}

For both VAN20 and VAN50 systems, 5000 steps of energy minimization were carried out, using the steepest descent method, after which the systems were simulated, for a total simulation time, including equilibration, of 320 ns. All systems were simulated at 1 bar pressure and a temperature of 
$300 \mathrm{~K}\left(26.85^{\circ} \mathrm{C}\right)$. Periodic boundary conditions were used in all directions. Berendsen thermostat and barostat [46] were used with coupling constants set to 0.5 and $5.01 / \mathrm{s}$ respectively. For the bulk PES system, an isotropic pressure coupling was employed, whereas, for the case of the PES-W, VAN20 and VAN50 systems, a semi-isotropic pressure coupling, with fixed X and Y dimensions, was used to maintain a stable PES-layer. Separate thermostats were used for the PES, vanillin and solvent molecules. Lennard-Jones interactions were cut-off at $1.0 \mathrm{~nm}$ and, for the electrostatic interactions, we employed the particle mesh Ewald method (PME) [47].

Analysis utilities found within the GROMACS (v. 5.0.4) suite [48] were used to determine the number of contacts as a function of time (gmx mindist), density profiles (gmx density) and surface tension (gmx energy). The number

of contacts between different molecules was defined with a distance cutoff of $0.6 \mathrm{~nm}$. For the mass density profiles, the last $200 \mathrm{~ns}$ were used for the data analysis and the Z-dimension of the simulation box was divided into 50 slices.

\section{Results and discussion}

\subsection{Raman spectroscopy: Adsorption of vanillin to the UH004 P PESH membrane}

The ATR-FTIR spectra of the membranes, after vanillin adsorption and flushing treatments, are shown in Fig. 3A. The highlighted peak at $1511 \mathrm{~cm}^{-1}$ is assigned to the skeletal vibrations of the aromatic ring of vanillin [49] and does not appear in the spectra of the virgin UH004 P membrane. Thus, the intensity of this peak can be seen as an indicator of the quantity of vanillin adsorbed onto the membrane; the ATR-FTIR spectroscopy result clearly shows that the concentration of vanillin adsorbed to the PES surface increases, as the $\mathrm{pH}$ of the adsorption conditions decreases and flushing causes significant desorption of vanillin from the membrane.

The quantity of vanillin on the flushed membranes was analyzed using the extraction and UV/Vis analysis methodologies. The measured concentrations are shown in Tab. S1, SM. Extraction results show low standard deviation (see SM, Tab. S1) and are within the detection range, despite the significant desorption of vanillin due to flushing; 81-92 \% of adsorbed vanillin desorbed from the membrane during flushing and pure water permeability measurement steps.

Average concentrations, calculated from the extraction data, were used to scale the concentration profiles. In all cases, the PCA score at the start- 

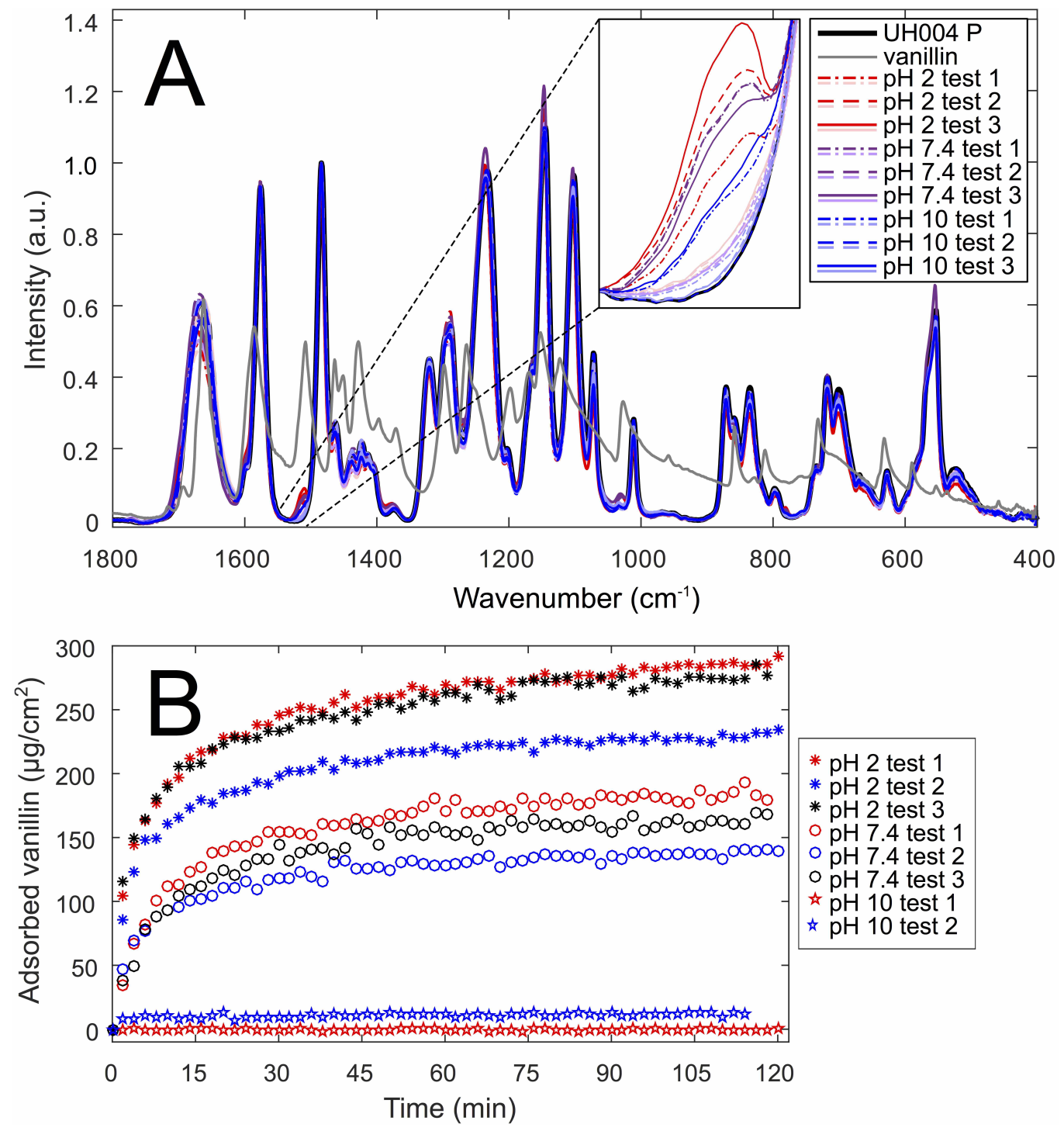

Figure 3: Spectroscopy results: A) ATR-FTIR spectra for vanillin, virgin UH004 P membrane, adsorption coated (bright lines) and flushed (pale lines) membranes. Adsorption conditions: $750 \mu \mathrm{g} / \mathrm{L}$ vanillin, $T=25{ }^{\circ} \mathrm{C}, t=120 \mathrm{~min}, v=0.4 \mathrm{~m} / \mathrm{s}, \mathrm{B}$ ) Scaled concentration profiles for experiments derived from PCA based models, calibrated using extraction and $\mathrm{UV} / \mathrm{Vis}$ analysis.

ing point of the concentration profiles was scaled to zero, since the initial condition was a clean PESH membrane immersed in a solution with vanillin present, i.e. no vanillin was adsorbed to the PESH surface at the start of 
the experiment. The final Raman spectra were measured after the flushing and measurement of the pure water permeability and immediately prior to removal of the membrane from the module; these results were used to define the scaling of the concentration profiles.

Scaled adsorption profiles, at different pH levels, are presented in Fig. 3B. It can be seen that the qualitative forms of the concentration profiles are very similar for the case of $\mathrm{pH}$ values of 2 and 7.4, however, quantitatively, we clearly see that more vanillin has adsorbed at $\mathrm{pH} 2$ than $\mathrm{pH}$ 7.4. The form of the concentration profile at $\mathrm{pH} \mathrm{10,} \mathrm{however,} \mathrm{does} \mathrm{not} \mathrm{resemble} \mathrm{that}$ at the other, lower, $\mathrm{pH}$ values; our interpretation of this result is that the quantity of adsorbed vanillin is below the detection limit of the apparatus. The Raman spectroscopy results thus show a clear picture: as $\mathrm{pH}$ decreases, vanillin adsorption to the PES surface increases.

\subsection{SPR: adsorption of vanillin to a PES coated gold sensor}

We measured an average SPR response, i.e. SPR peak angular shift, of $3.12 \pm 0.39^{\circ}$ (for wavelength $785 \mathrm{~nm}$ ) relative to the pure SPR sensor chip immersed in water. The average SPR responses, for each concentration and $\mathrm{pH}$ value, are shown in Fig. 4. In all cases the form of the response curve indicates a rapid initial binding event, possibly the aggregation of vanillin to the rough PES surface, followed by a slower accumulation phase. Additionally, we see a clear trend: a decrease in the adsorption of vanillin onto the PES surface with increase in $\mathrm{pH}$. The lowest concentration $(25 \mu \mathrm{g} / \mathrm{mL})$ proved insufficient to induce binding to the PES surface at $\mathrm{pH} 10$, thus results for this concentration and $\mathrm{pH}$ are omitted.

As stated in section 2.3.4, our results for the binding kinetics were successfully fit to a one-to-two (1:2) binding model: adsorption governed by two separate mechanisms. As we have postulated in the introduction, we expect both electrostatic and entropy driven effects to govern the adsorption of vanillin to the PES surface: two mechanisms; this is not an unexpected outcome. In Tab. 2, we show the fitted one-to-two model dissociation constants, at different $\mathrm{pH}$ values; both dissociation constants increase with with increasing $\mathrm{pH}$ : there is a negative correlation between $\mathrm{pH}$ and the affinity of vanillin for the PES membrane.

The total internal reflection (TIR) angle in SPR measurements also affects the SPR peak angular position; its contribution to the SPR response curves was removed from the association kinetic curves to obtain the above kinetic analysis utilizing the PureKinetics ${ }^{T M}$ analysis software tool (Data Viewer 

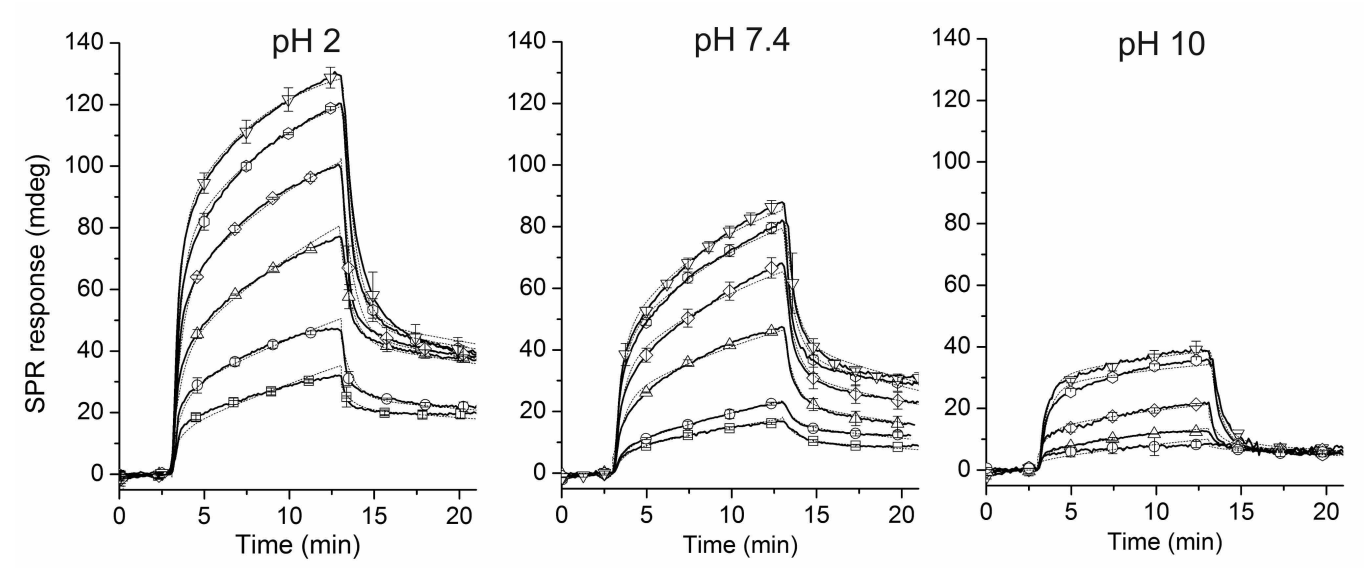

Figure 4: SPR results: responses (solid lines) at different $\mathrm{pH}$ values for vanillin concentrations $25(\square), 75(\circ), 225(\triangle), 450(\diamond), 675(\bigcirc)$ and $1000(\nabla) \mu \mathrm{g} / \mathrm{mL}$. Dashed lines depict the fitted curves from one-to-two kinetic model data fitting. The standard deviation for all results is shown.

Table 2: One-to-two model dissociation constants derived from curve fitting at different $\mathrm{pH}$ levels with the respective $\chi^{2}$-values for each of the three fits.

\begin{tabular}{llll}
\hline $\mathrm{pH}$ & $K_{D 1}(\mathrm{mM})$ & $K_{D 2}(\mathrm{mM})$ & Chi-Square $\left(\chi^{2}\right)$ \\
\hline 2 & $0.82 \pm 0.07$ & $7.380 \pm 0.007$ & 2.45 \\
\hline 7.4 & $3.52 \pm 0.10$ & $15.60 \pm 0.02$ & 1.60 \\
\hline 10 & $7.27 \pm 0.73$ & $22.5 \pm 0.1$ & 0.81 \\
\hline
\end{tabular}

Software v. 4.2.4, BioNavis Ltd., Tampere, Finland) provided with the MPSPR instrument. The TIR angle, however, itself, provides valuable insight regarding the properties of the bulk solution phase; a shift in the TIR angle reflects a change in the refractive index of the bulk liquid. In our case, this change results from the entry of vanillin into the bulk phase; the extent of the TIR shift can be seen as a measure of the quantity of vanillin in the bulk liquid phase, rather than at the interface. At both $\mathrm{pH} 2$ and 7.4, this shift was only observed for the system with the highest vanillin concentration $(1 \mathrm{mg} / \mathrm{mL})$. At $\mathrm{pH} 10$, in addition to the highest concentration, TIR shifts were also observed at vanillin concentrations of 450 and $675 \mu \mathrm{g} / \mathrm{mL}$. For a concentration of $1 \mathrm{mg} / \mathrm{mL}$, the average shifts in TIR angle were calculated as $15.9 \pm 2.0(\mathrm{pH} 2), 18.4 \pm 3.7(\mathrm{pH} 7.4)$ and $23.0 \pm 2.3 \operatorname{mdeg}(\mathrm{pH} 10)$. 
Overall, it can clearly be seen that, at higher $\mathrm{pH}$, the quantity of vanillin in solution is increased; this provides further evidence that the adsorption of vanillin to the PES membrane decreases with increasing $\mathrm{pH}$.

\subsection{MD simulation: mechanism of vanillin adsorption to the PES surface}

We have now established, through both Raman Spectroscopy and Surface Plasmon resonance that a strong $\mathrm{pH}$ dependence exists regarding the affinity of vanillin for the $\mathrm{PES}(\mathrm{H})$ membrane. It is also known that, in acidic $\mathrm{pH}$, the hydroxyl group of vanillin is predominantly protonated, while most vanillin molecules are unprotonated above $\mathrm{pH}$ 7.4. Thus, through computational MD simulation, of both protonated and unprotonated vanillin molecules interacting with the PES surface, we can determine the mechanism of this $\mathrm{pH}$ dependence. To recap, two systems were simulated, a PES layer interacting with 1) 20 vanillin molecules, 10 protonated and 10 not protonated: "VAN20" and 2) 50 protonated vanillin molecules: "VAN50". All simulation parameters are described in the materials and methods section (Section 2.4).

The number of contacts per atom, as a function of time, both vanillinvanillin and PES-vanillin, was measured for both systems; plots of this data are found in SM, see Fig. S3. While, regarding these quantities, the equilibrium values are reached extremely rapidly for the Van20 system, equilibration takes slightly longer for the Van50 system. Interestingly, in the Van50 system, the equilibration processes, for the number of vanillin-vanillin and PES-vanillin contacts, take place on different time scales; the number of PESvanillin contacts increases, to reach an equilibrium value at $\sim 15 \mathrm{~ns}$, of almost complete association, i.e. a value of unity, while the number of vanillinvanillin contacts decreased, to reach an equilibrium value at $\sim 50$ ns. This indicates that the vanillin molecules are still dissociating from each other once they have equilibrated to the surface. From the plot of the Van20 system, it can clearly be seen that virtually all the protonated vanillin molecules are always associated with the PES surface, while the number of unprotonated vanillin molecules that are not associated, fluctuates in the range $0-20 \%$. The number of contacts between protonated vanillin molecules is greater than that between the unprotonated vanillin molecules; this is a result of coulombic repulsion, due to the charged nature of unprotonated vanillin.

To obtain a quantitative comparison, of the different behavior of the protonated and unprotonated forms of vanillin, at the PES surface, we calculated the mass density profile of water, PES and vanillin molecules for both systems. The mass density profile is a measure of the number of a 
specific atom, atom group, or entire molecules located at specific positions normal to the plane of the PES surface, i.e. where in the PES surface the molecule sits. As shown in Fig. 5A, the protonated vanillin molecules have penetrated considerably deeper into the PES layer than the unprotonated vanillin; visualizations of the two systems, again, see Fig. 5A, also show this: the protonated vanillin is entirely within the PES layer, embedded in the surface, while some of the unprotonated vanillin is in solution.

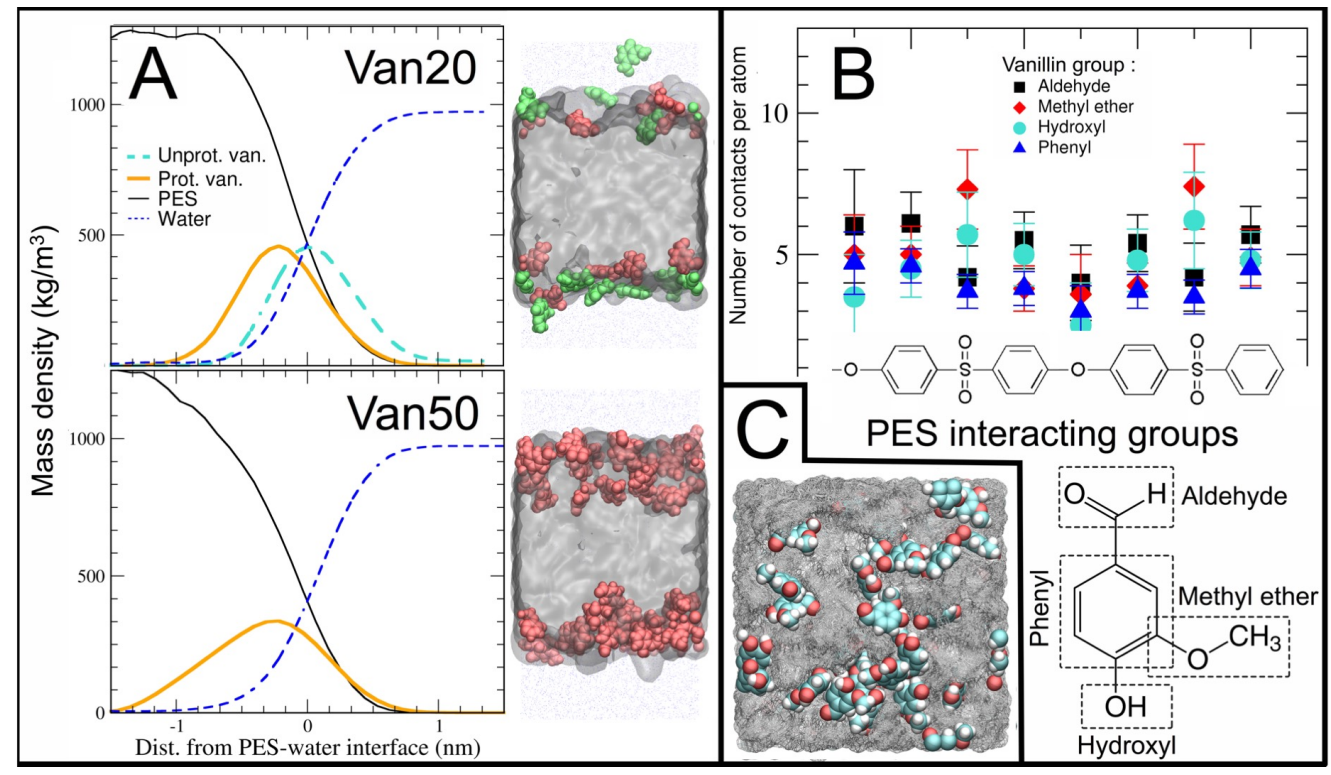

Figure 5: MD simulation results: A) Mass density profiles for VAN20 and VAN50 systems, with snapshots from the end of both simulations. For the case of the VAN20 system, the densities of unprotonated and protonated vanillin were multiplied by five for clarity. In the snapshots, the water molecules are rendered as blue dots, PES molecules are grey van der Waals spheres, protonated and unprotonated vanillin molecules are red and green van der Waals spheres respectively, B) The number of contacts per atom between the different Vanillin and PES groups, C) Snapshot of the PES surface showing deposited protonated vanillin molecules.

Now that we have established that the protonated form of vanillin has the stronger interaction with the PES surface, we proceed to investigate the mechanism behind this. We first measured, shown in Fig. 5B, the number of contacts per atom between the different interacting groups of PES and the four different groups of the vanillin molecule: aldehyde, methyl ether, hydroxyl and phenyl (see Fig. 5B). It is apparent that there is increased contact between the sulphur oxygen group of PES and the hydroxyl and methyl 
ether groups of vanillin. The number of H-bonds between the hydroxyl group of vanillin and both $\mathrm{H}$-bond acceptor sites on the PES, the ether and sulphur oxygens, was then determined; an H-bond was considered to be formed when the distance between donor and acceptor was $0.35 \mathrm{~nm}$ or less and the hydrogen-donor-acceptor angle was 30 degrees or less; this is a commonly accepted definition of an H-bond in the context of MD simulation with all atom resolution [50-52]. We found instances of H-bond formation between the hydroxyl group of vanillin and both the sulphur and ether oxygens, however H-bonding to the sulphur oxygens was clearly the dominant mechanism of the two; $98 \%$ and $99 \%$ of H-bonds were with the sulphur oxygen in the Van20 and Van50 systems respectively. While the extent to which H-bonding occurred was significant, it was not the dominant mechanism of interaction between vanillin and the PES surface; in both cases a minority of the protonated vanillin molecules were H-bonded to PES: $16 \pm 10 \%$ and $21 \pm 5 \%$ for the Van20 and Van50 systems, respectively; the dominant mechanism for the binding of protonated vanillin to the PES surface is thus hydrophobic (entropy driven) interactions between the phenyl rings of PES and vanillin.

\section{Conclusion}

The results from our separate experimental protocols, SPR and Raman and ATR-FTIR spectroscopy, all clearly show a strong $\mathrm{pH}$ dependence on the affinity of the PES surface for vanillin: affinity is greatly enhanced as $\mathrm{pH}$ is reduced. Additionally, the SPR results indicated that the quantity of vanillin in solution increased with increasing $\mathrm{pH}$. The $\mathrm{SPR}$ results also provide insight into the binding kinetics: 1) two separate binding mechanisms are seen to be present, one clearly stronger than the other that 2) both become stronger with decreasing $\mathrm{pH}$ and 3) in all cases the form of the response curve indicates a rapid initial binding event followed by a slower accumulation phase.

MD simulation provides the mechanism behind these observed phenomena: 1) As $\mathrm{pH}$ increases, a greater proportion of vanillin is in the unprotonated form that has a weaker interaction with the PES surface; 2) the unprotonated form of vanillin has a greater water solubility, thus explaining the result of the increased quantity of solvated vanillin at higher $\mathrm{pH}$; 3) the two separate binding mechanisms, identified by SPR measurements, are a dominant mechanism due to entropy driven, hydrophobic, interactions between phenyl rings of PES and vanillin and a weaker mechanism due to Hbonding, predominantly between the hydroxyl group of the protonated form 
of vanillin and the sulphur oxygens of PES.

In the MD simulation, we noted an increased degree of contact between the sulphur oxygens of PES and the methyl ether group, in addition to the hydroxyl group of vanillin: this can be attributed to the close proximity of the two groups on the vanillin molecule. The SPR result, of an initial rapid binding followed by a slow accumulation phase, can be related to the MD results that show the adsorption to the PES surface occurring on a faster timescale than the dissociation of vanillin molecules from each other, through the following picture: the binding due to hydrophobic effects occurs on a rapid timescale, as this is the dominant mechanism; on a slower timescale, the vanillin molecules dissociate from each other as they H-bond to the sulphur oxygens of the PES, spreading out to form a more even coating on the PES surface; the vanillin molecules actually disperse, rather than aggregate, on the PES surface. The MD results also showed that protonated vanillin penetrates deeper into the PES matrix than unprotonated vanillin; this is clearly due to both its more hydrophobic nature and the extra H-bond that draws it into the PES surface.

The increase in both dissociation constants that corresponds to decreased binding, measured by SPR, indicates that, as $\mathrm{pH}$ rises, both the H-bonding and hydrophobic, entropy driven, interactions become reduced. This is explained by the decreasing portion of protonated vanillin that is present and the more hydrophilic nature of unprotonated vanillin. There is, however, another factor at work: it has been shown by Lin et al. [53] that the UH004 P membrane shows amphoteric behavior as a function of $\mathrm{pH}$, determined from the dissociation of the functional groups present on the active layer of the membrane [54]; At low $\mathrm{pH}$, the membrane has a lower degree of negative charge, in comparison to the case at neutral and high $\mathrm{pH}$, where the surface of the membrane is mostly negatively charged: at high $\mathrm{pH}$ both the, now unprotonated, vanillin molecules and PES membrane will be negatively charged, thus repel each other to an even greater extent.

We now have a mechanistic understanding of vanillin adsorption to a PES surface and how this interaction is dependent on $\mathrm{pH}$, shown in Fig. 6; so, how can we now apply this knowledge to the more general area of the development of strategies to control (minimize) absorptive fouling to the skin layer of a membrane? Basically, now that we know what the game is, we can play it: the key interacting elements of phenolic compounds that initiate binding to the PES surface, are hydrophobic phenyl groups and hydroxyl groups that are H-bond donors, capable of forming H-bonds with the sulphur oxygens that 
act as H-bond acceptors. This interaction is highly dependent on the pKa of the specific hydroxyl group. Functionalization of the sulphur group, with a relatively hydrophilic side chain, but with weaker H-bond acceptor sites, may lower adsorptive fouling. Molecular level knowledge about hydrogen bonding and adsorptive interactions can be also exploited to confer selectivity in adsorptive separations[55].

\section{$\mathrm{pH} 2$}

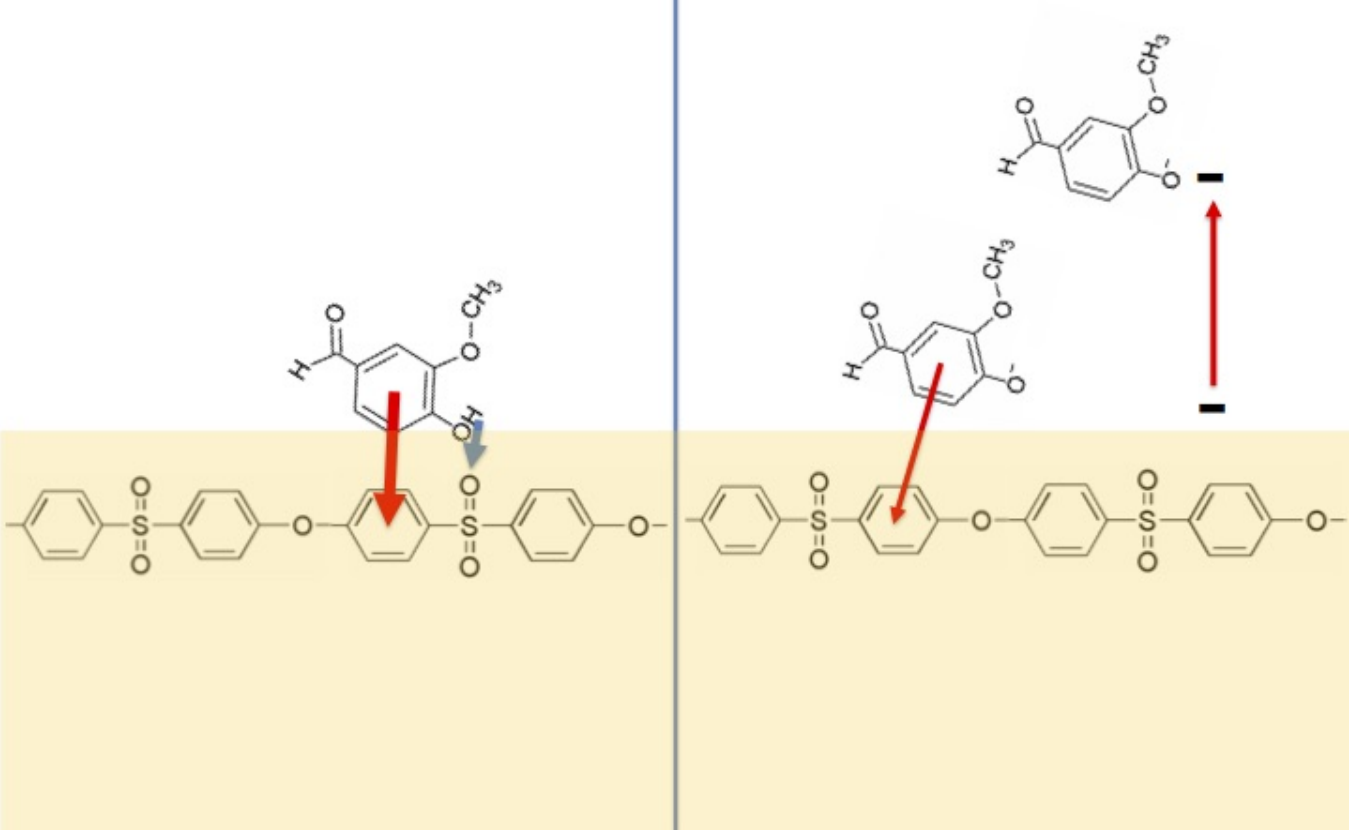

Figure 6: Mechanism of $\mathrm{pH}$ dependence of vanillin interaction with the PES surface: at low $\mathrm{pH}$ the molecule, in its protonated form, is more hydrophobic, thus has a stronger hydrophobic (entropic) interaction and is additionally able to H-bond to the sulphur oxygens of the PES; at high pH both PES and vanillin are negatively charged, resulting in a repulsive interaction that decreases binding still further.

In the context of the potential role of vanillin as an antifouling coating, anti-fouling molecules, based on the structure of vanillin, can be developed; the molecular structure can be adjusted to alter the pKa of the hydroxyl group, to perform over a different $\mathrm{pH}$ range, if desired. Information on interactions between vanillin and polyethersulfone can be also exploited in 
development of controlled release materials, such as microcapsules [56, 57]. Our computational results also indicate that vanillin molecules continue to dissociate from each other once they have reached the surface, indicating the potential to evenly coat the PES surface; this behavior possibly includes the case of a highly porous PES membrane. In designing new molecules for antifouling coatings based on vanillin, this property could possibly be still further enhanced.

Our results, regarding the interaction of vanillin with a PES membrane, provide more general insight into the adsorptive tendencies of all phenolic and ligneous compounds. This can be used to develop alterations to the physicochemical properties of the phenolic compounds, for instance via catalysis, that could lead to a decrease in the extent of adsorptive fouling. All processes where phenolic and ligneous materials are present are affected by this; this information can be used in the development of means to reduce fouling problems on any surface. All of this proposed development work can be performed using the new synergistic protocol we have developed that combines experimental methodologies, spectroscopy and SPR with MD simulation; we propose that this protocol can be used even more broadly in this area of research.

Our study assists in the development of methods to both control membrane fouling by phenolic compounds and to engineer novel antifouling surfaces and adsorption resins [58-60] through the controlled adsorption of phenolic compounds. In a more general context, our insight can be applied to a broader range of issues related to the filtration of water that contains phenolic or ligneous compounds. In addition, this piece of work also represents a methodologically novel combined protocol for the analysis of a wide range of systems involving adsorption to a surface.

\section{Acknowledgements}

The authors would like to thank Eija Saski for discussions. This research was funded by the Academy of Finland (Grant No. 292253 and No. 288619). The computational work carried out using the resources of the CSC-IT Centre for Science Ltd. (CSC). We also express special thanks to Xue Zhang and KSV-NIMA for collaborating with the AFM and PM-IRRAS experiments, respectively. 


\section{References}

[1] J. A. Koehler, M. Ulbricht, G. Belfort, Intermolecular forces between a protein and a hydrophilic modified polysulfone film with relevance to filtration, Langmuir 16 (26) (2000) 10419-10427.

[2] T. Dey, Nanotechnology for Water Purification, Universal Publishers Inc., Boca Raton, 2012.

[3] P. Xiao, F. Xiao, D. sheng Wang, T. Qin, S. ping He, Investigation of organic foulants behavior on hollow-fiber UF membranes in a drinking water treatment plant, Sep. Purif. Technol. 95 (2012) 109-117.

[4] L. Ao, W. Liu, L. Zhao, X. Wang, Membrane fouling in ultrafiltration of natural water after pretreatment to different extents, J. Environ. Sci. 43 (2016) 234-243.

[5] C. M. Chew, M. K. Aroua, M. A. Hussain, A practical hybrid modelling approach for the prediction of potential fouling parameters in ultrafiltration membrane water treatment plant, J. Ind. Eng. Chem. 45 (2017) $145-155$.

[6] B. K. Pramanik, A. Kajol, F. Suja, S. M. Zain, Effect of biological and coagulation pre-treatments to control organic and biofouling potential components of ultrafiltration membrane in the treatment of lake water, Environ. Technol. 38 (5) (2017) 579-587.

[7] E. Akhondi, F. Zamani, K. H. Tng, G. Leslie, W. B. Krantz, A. G. Fane, J. W. Chew, The performance and fouling control of submerged hollow fiber (HF) systems: A review, Appl. Sci. 7 (2017) 765.

[8] C. Zhao, J. Xue, F. Ran, S. Sun, Modification of polyethersulfone membranes - a review of methods, Prog. Mater. Sci. 58 (1) (2013) 76-150.

[9] A. Figoli, A. Cassano, A. Basile, Membrane Technologies for Biorefining, Elsevier Science, 2016.

[10] N. L. Le, S. P. Nunes, Materials and membrane technologies for water and energy sustainability, Sustainable Mater. Technol. 7 (2016) 1-28. 
[11] H. Li, P. Yu, Y. Luo, Correlation between organic fouling of reverseosmosis membranes and various interfacial interactions, Chem. Eng. Technol. 38 (1) (2015) 131-138.

[12] A. I. Schäfer, I. Akanyeti, A. J. Semião, Micropollutant sorption to membrane polymers: A review of mechanisms for estrogens, Adv. Colloid Interfac. 164 (1-2) (2011) 100-117.

[13] L. Braeken, R. Ramaekers, Y. Zhang, G. Maes, B. V. der Bruggen, C. Vandecasteele, Influence of hydrophobicity on retention in nanofiltration of aqueous solutions containing organic compounds, J. Membr. Sci. 252 (1-2) (2005) 195-203.

[14] E. Koivula, M. Kallioinen, S. Preis, L. Testova, H. Sixta, M. Mänttäri, Evaluation of various pretreatment methods to manage fouling in ultrafiltration of wood hydrolysates, Sep. Purif. Technol. 83 (2011) 50-56.

[15] E. Koivula, M. Kallioinen, T. Sainio, E. Antón, S. Luque, M. Mänttäri, Enhanced membrane filtration of wood hydrolysates for hemicelluloses recovery by pretreatment with polymeric adsorbents, Bioresour. Technol. 143 (2013) 275-281.

[16] A. Maartens, E. Jacobs, P. Swart, UF of pulp and paper effluent: membrane fouling-prevention and cleaning, J. Membr. Sci. 209 (1) (2002) 81-92.

[17] W. Yuan, A. L. Zydney, Humic acid fouling during ultrafiltration, Environ. Sci. Technol. 34 (23) (2000) 5043-5050.

[18] R. Sierra-Alverez, G. Lettinga, The methogenic toxicity of wastewater lignins and lignin related gompounds, J. Chem. Tech. Biotechnol. 50 (4) (1991) 443-455.

[19] D. Pokherel, T. Viraraghavan, Treatment of pulp and paper mill wastewater - a review, Sci. Total Environ. 333 (1) (2004) 37-58.

[20] T. Kreetachat, M. Damrongsri, V. Punsuwon, P. Vaithanomsat, C. Chiemchaisri, C. Chomsurin, Effects of ozonation process on ligninderived compounds in pulp and paper mill effluents, J. Hazard Mater. $142(1-2)(2007)$ 250-257. 
[21] M. N. De Pinho, M. Minhalma, M. J. Rosa, F. Taborda, Integration of flotation/ultrafiltration for treatment of bleached pulp effluent: helps to remove suspended solids and color, Pulp Pap. Can. 101 (4) (2000) $50-54$.

[22] H. Susanto, Y. Feng, M. Ulbricht, Fouling behavior of aqueous solutions of polyphenolic compounds during ultrafiltration, J. Food Eng. 91 (2) (2009) 333-340.

[23] J. Luo, B. Zeuner, S. T. Morthensen, A. S. Meyer, M. Pinelo, Separation of phenolic acids from monosaccharides by low-pressure nanofiltration integrated with laccase pre-treatments, J. Membr. Sci. 482 (2015) 83-91.

[24] A. Cassano, E. Drioli, Integrated Membrane Operations: In the Food Production, De Gruyter, 2014.

[25] K. Ponnusamy, D. Paul, J. H. Kweon, Inhibition of quorum sensing mechanism and Aeromonas hydrophila biofilm formation by vanillin, Environ. Eng. Sci. 26 (8) (2009) 1359-1363.

[26] S. Kappachery, D. Paul, J. Yoon, J. H. Kweon, Vanillin, a potential agent to prevent biofouling of reverse osmosis membrane, Biofouling 26 (6) (2010) 667-672.

[27] K. Ponnusamy, S. Kappachery, M. Thekeettle, J. H. Song, J. H. Kweon, Anti-biofouling property of vanillin on Aeromonas hydrophila initial biofilm on various membrane surfaces, World J. Microbiol. Biotechnol. 29 (9) (2013) 1695-1703.

[28] A. Nam, J. Kweon, J. Ryu, H. Lade, C. Lee, Reduction of biofouling using vanillin as a quorum sensing inhibitory agent in membrane bioreactors for wastewater treatment, Membr. Water Treat. 6 (3) (2015) 189203.

[29] E. Smith, G. Dent, Modern Raman Spectroscopy: A Practical Approach, Wiley, Chichester, 2005.

[30] L. G. Thygesen, M. M. Løkke, E. Micklander, S. B. Engelsen, Vibrational microspectroscopy of food. Raman vs. FT-IR, Trends Food Sci. Technol. 14 (1-2) (2003) 50-57. 
[31] T. Virtanen, S.-P. Reinikainen, M. Kögler, M. Kallioinen, T. Viitala, M. Mänttäri, Real-time fouling monitoring with Raman spectroscopy, J. Membr. Sci. 525 (2017) 312-319.

[32] B. Liedberg, C. Nylander, I. Lunström, Surface plasmon resonance for gas detection and biosensing, Sens. Actuators 4 (1983) 299-304.

[33] B. Liedberg, C. Nylander, I. Lunström, Biosensing with surface plasmon resonance - how it all started, Biosens. Bioelectron. 10 (8) (1995) i-ix.

[34] H. H. Nguyen, J. Park, S. Kang, M. Kim, Surface plasmon resonance: a versatile technique for biosensor applications, Sensors 15 (5) (2015) 10481-10510.

[35] E. H. Lee, J. Hsin, M. Sotomayor, G. Comellas, K. Schulten, Discovery through the computational microscope, Structure 17 (10) (2009) 12951306.

[36] Open Notebook Science Challenge: Exp018 (September 2017).

[37] J. Kim, N. Weber, G. Shin, Q. Huang, S. Liu, The study of $\beta$ lactoglobulin adsorption on polyethersulfone thin film surface using QCM-D and AFM, J. Food Sci. 72 (4) (2007) E214-E221.

[38] R. Karlsson, A. Fält, Experimental design for kinetic analysis of proteinprotein interactions with surface plasmon resonance biosensors, J. Immunol. Methods 200 (1) (1997) 121-133.

[39] W. L. Jorgensen, J. Tirado-Rives, The OPLS potential functions for proteins, energy minimizations for crystals of cyclic peptides and crambin, J. Am. Chem. Soc. 110 (6) (1988) 1657-1666.

[40] W. L. Jorgensen, J. Chandrasekhar, J. D. Madura, R. W. Impey, M. L. Klein, Comparison of simple potential functions for simulating liquid water, J. Chem. Pys. 79 (2) (1983) 926-935.

[41] M. J. Frisch, G. W. Trucks, G. E. Schlegel, H. B.and Scuseria, Gaussian09 Revision E.01Gaussian Inc. Wallingford CT 2009.

[42] D. A. Case, R. M. Betz, W. Botello-Smith, D. S. Certulli, T. E. Cheatham III, T. A. Darden, R. E. Duke, T. J. Giese, H. Gohlke, 
A. W. Goetz, N. Homeyer, S. Izadi, P. Janowski, J. Kaus, A. Kovalenko, T. S. Lee, S. LeGrand, P. Li, C. Lin, T. Luchko, R. Luo, B. Madej, D. Mermelstein, K. M. Merz, G. Monard, H. Nguyen, H. T. Nguyen, I. Omelyan, A. Onufriev, D. R. Roe, A. Roitberg, C. Sagui, C. L. Simmerling, J. Swails, R. C. Walker, J. Wang, R. Wolf, X. Wu, L. Xiao, D. M. York, P. A. Kollman, AMBER 2016, University of California, San Francisco (2016).

[43] U. C. Singh, P. A. Kollman, An approach to computing electrostatic charges for molecules, J. Comput. Chem. 5 (2) (1984) 129-145.

[44] C. I. Bayly, P. Cieplak, W. Cornell, P. A. Kollman, A well-behaved electrostatic potential based method using charge restraints for deriving atomic charges: the RESP model, J. Phys. Chem. 97 (40) (1993) 1026910280 .

[45] A. A. S. T. Ribeiro, B. A. C. Horta, R. B. d. Alencastro, MKTOP: a program for automatic construction of molecular topologies, J. Braz. Chem. Soc. 19 (7) (2008) 1433-1435.

[46] H. J. C. Berendsen, J. P. M. Postma, W. F. van Gunsteren, A. DiNola, J. R. Haak, Molecular dynamics with coupling to an external bath, J. Chem. Phys. 81 (8) (1984) 3684-3690.

[47] U. Essmann, L. Perera, M. L. Berkowitz, T. Darden, H. Lee, L. G. Pedersen, A smooth particle mesh Ewald method, J. Chem. Phys. 103 (19) (1995) 8577-8593.

[48] H. Berendsen, D. van der Spoel, R. van Drunen, GROMACS: A messagepassing parallel molecular dynamics implementation, Comput. Phys. Commun. 91 (1) (1995) 43-56.

[49] V. Balachandran, K. Parimala, Vanillin and isovanillin: Comparative vibrational spectroscopic studies, conformational stability and NLO properties by density functional theory calculations, Spectrochim. Acta, Part A 95 (2012) 354-368.

[50] K. Murzyn, T. Róg, G. Jezierski, Y. Takaoka, M. Pasenkiewicz-Gierula, Effects of phospholipid unsaturation on the membrane/water interface: a molecular simulation study, Biophys. J. 81 (1) (2001) 170-183. 
[51] K. Modig, B. G. Pfrommer, B. Halle, Temperature-dependent hydrogenbond geometry in liquid water, Phys. Rev. Lett. 90 (7) (2003) 075502.

[52] D. van der Spoel, P. J. van Maarten, N. Tîmneanu, Thermodynamics of hydrogen bonding in hydrophilic and hydrophobic media, J. Phys. Chem. B 110 (9) (2006) 4393-4398.

[53] J. Lin, W. Ye, M.-C. Baltaru, Y. P. Tang, N. J. Bernstein, P. Gao, S. Balta, M. Vlad, A. Volodin, A. Sotto, P. Luis, A. L. Zydney, B. V. der Bruggen, Tight ultrafiltration membranes for enhanced separation of dyes and Na2SO4 during textile wastewater treatment, J. Membr. Sci. 514 (2016) 217-228.

[54] S. Bandini, C. Mazzoni, Modelling the amphoteric behaviour of polyamide nanofiltration membranes, Desalination 184 (1) (2005) 327336 .

[55] A. J. Glemza, K. L. Mardis, A. A. Chaudhry, M. K. Gilson, G. F. Payne, Competition between intra- and intermolecular hydrogen bonding:? effect on para/ortho adsorptive selectivity for substituted phenols, Industrial \& Engineering Chemistry Research 39 (2) (2000) 463-472.

[56] C. Panisello, B. Peña, G. Gilabert Oriol, M. Constantí, T. Gumí, R. Garcia-Valls, Polysulfone/vanillin microcapsules for antibacterial and aromatic finishing of fabrics, Industrial \& Engineering Chemistry Research 52 (29) (2013) 9995-10003.

[57] B. Pena, L.-C. de Menorval, R. Garcia-Valls, T. Gumi, Characterization of polysulfone and polysulfone/vanillin microcapsules by H-1 NMR ppectroscopy, solid-state C-13 CP/MAS-NMR spectroscopy, and N-2 adsorption desorption analyses, ACS Applied Materials \& Interfaces 3 (11) (2011) 4420-4430.

[58] M. I. F. Mota, P. C. R. Pinto, J. M. Loureiro, A. E. Rodrigues, Adsorption of vanillin and syringaldehyde onto a macroporous polymeric resin, Chemical Engineering Journal 288 (2016) 869-879.

[59] C. Michailof, G. G. Stavropoulos, C. Panayiotou, Enhanced adsorption of phenolic compounds, commonly encountered in olive mill wastewaters, on olive husk derived activated carbons, Bioresource Technology 99 (14) (2008) 6400-6408. 
[60] S. Mondal, R. Mukherjee, S. De, Process modeling for the removal of phenolic compounds from industrial wastewater using a mixed-matrix membrane, Industrial \& Engineering Chemistry Research 54 (1) (2015) $514-521$. 
Supplementary Material

S.1 
Table S1: Results from extraction and UV/vis analysis of flushed membrane samples.

\begin{tabular}{|c|c|c|c|c|c|c|}
\hline \multirow[b]{2}{*}{ Cell } & \multirow[b]{2}{*}{ Sample } & \multirow[b]{2}{*}{ Test } & \multicolumn{4}{|c|}{ Measured concentration } \\
\hline & & & $\begin{array}{l}\text { Piece } 1 \\
\left(\mu \mathrm{g} / \mathrm{cm}^{2}\right)\end{array}$ & $\begin{array}{l}\text { Piece } 2 \\
\left(\mu \mathrm{g} / \mathrm{cm}^{2}\right)\end{array}$ & $\begin{array}{l}\text { Piece } 3 \\
\left(\mu \mathrm{g} / \mathrm{cm}^{2}\right)\end{array}$ & $\begin{array}{l}\text { Average } c \\
\left(\mu \mathrm{g} / \mathrm{cm}^{2}\right)\end{array}$ \\
\hline \multirow{3}{*}{$\mathrm{A}$} & \multirow{3}{*}{$\mathrm{pH}$ 2, flushed } & $1 / 3$ & 32.8 & 35.1 & 37.4 & $35.1 \pm 1.4$ \\
\hline & & $2 / 3$ & 31.7 & 33.1 & 34.8 & $33.2 \pm 0.90$ \\
\hline & & $3 / 3$ & 29.4 & 17.4 & 16.9 & $21.2 \pm 4.1$ \\
\hline \multirow{3}{*}{$\mathrm{A}$} & \multirow{3}{*}{$\mathrm{pH} 7.4$, flushed } & $1 / 3$ & 24.1 & 21.9 & 18.9 & $21.7 \pm 1.6$ \\
\hline & & $2 / 3$ & 20.9 & 23.0 & 24.6 & $22.8 \pm 1.1$ \\
\hline & & $3 / 3$ & 33.7 & 29.7 & 31.5 & $31.6 \pm 1.2$ \\
\hline \multirow[b]{2}{*}{$\mathrm{A}$} & \multirow[b]{2}{*}{ pH 10, flushed } & $1 / 2$ & 5.5 & 6.0 & 5.7 & $5.7 \pm 0.16$ \\
\hline & & $2 / 2$ & 6.5 & 6.3 & 5.7 & $6.1 \pm 0.26$ \\
\hline
\end{tabular}



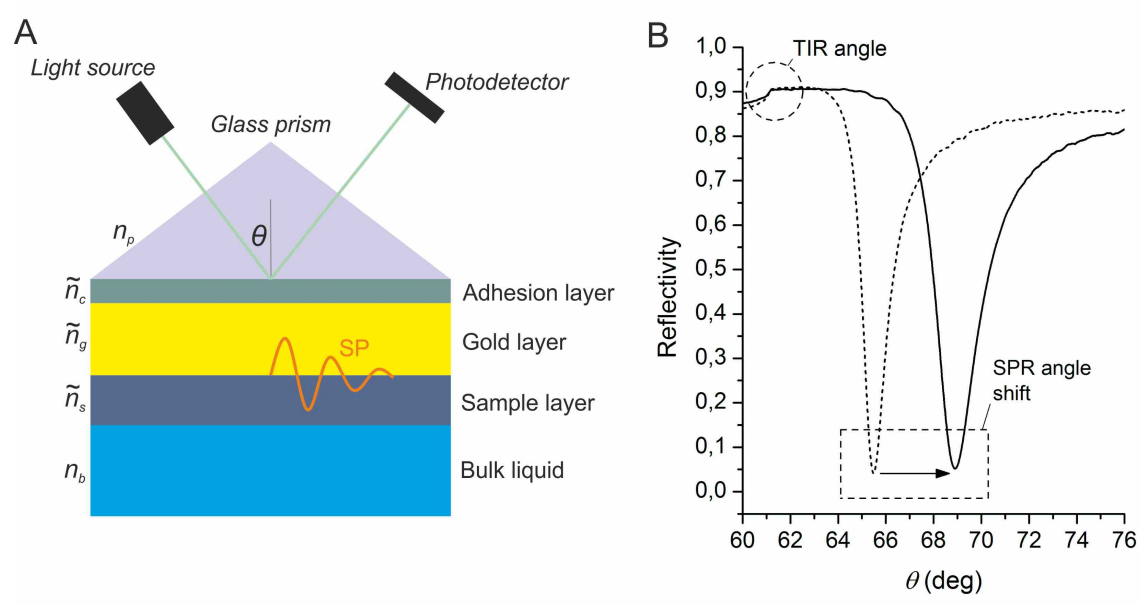

Figure S1: A simplified depiction of a multi-parametric surface plasmon resonance (MPSPR) experiment in the Kretschmann configuration. A laser source casts an incident beam (monochromatic p-polarized) with varying laser angle $\theta$ onto the sensor surface that induces the electrons in the gold atoms to form periodic oscillations on the surface, known as surface plasmons (SP). B) A typical reflectivity curve obtained from a single MPSPR scan. At a certain incident angle, the resonance angle, the optical energy becomes coupled and most photons are converted into surface plasmons. This results in a dip in the reflected light intensity measured as a function of the incident angle. Very small changes, in either the refractive index or thickness of the film attached to the gold surface, will cause a measurable deflection to this angle, known as $S P R$ peak angular shift. In addition, MP-SPR is capable to measure the angle of total internal reflection (TIR). 


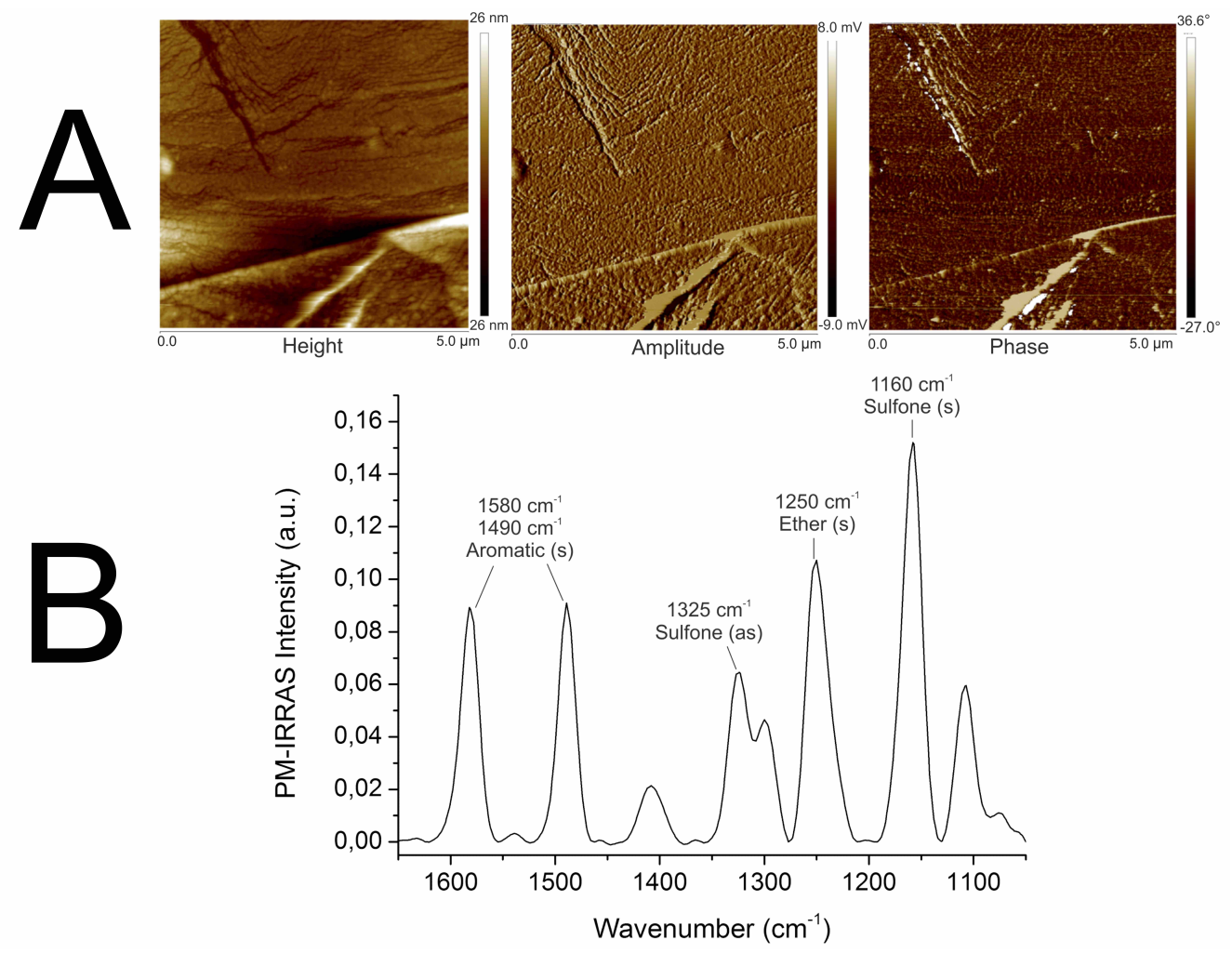

Figure S2: A) AFM height distribution, amplitude and phase of the PES-coated gold sensor, B) Baseline-corrected PM-IRRAS spectrum of the PES-coated gold sensor surface showing characteristic peaks for polyethersulfone ( $\mathrm{s}=$ stretch, as = antisymmetric stretch). 


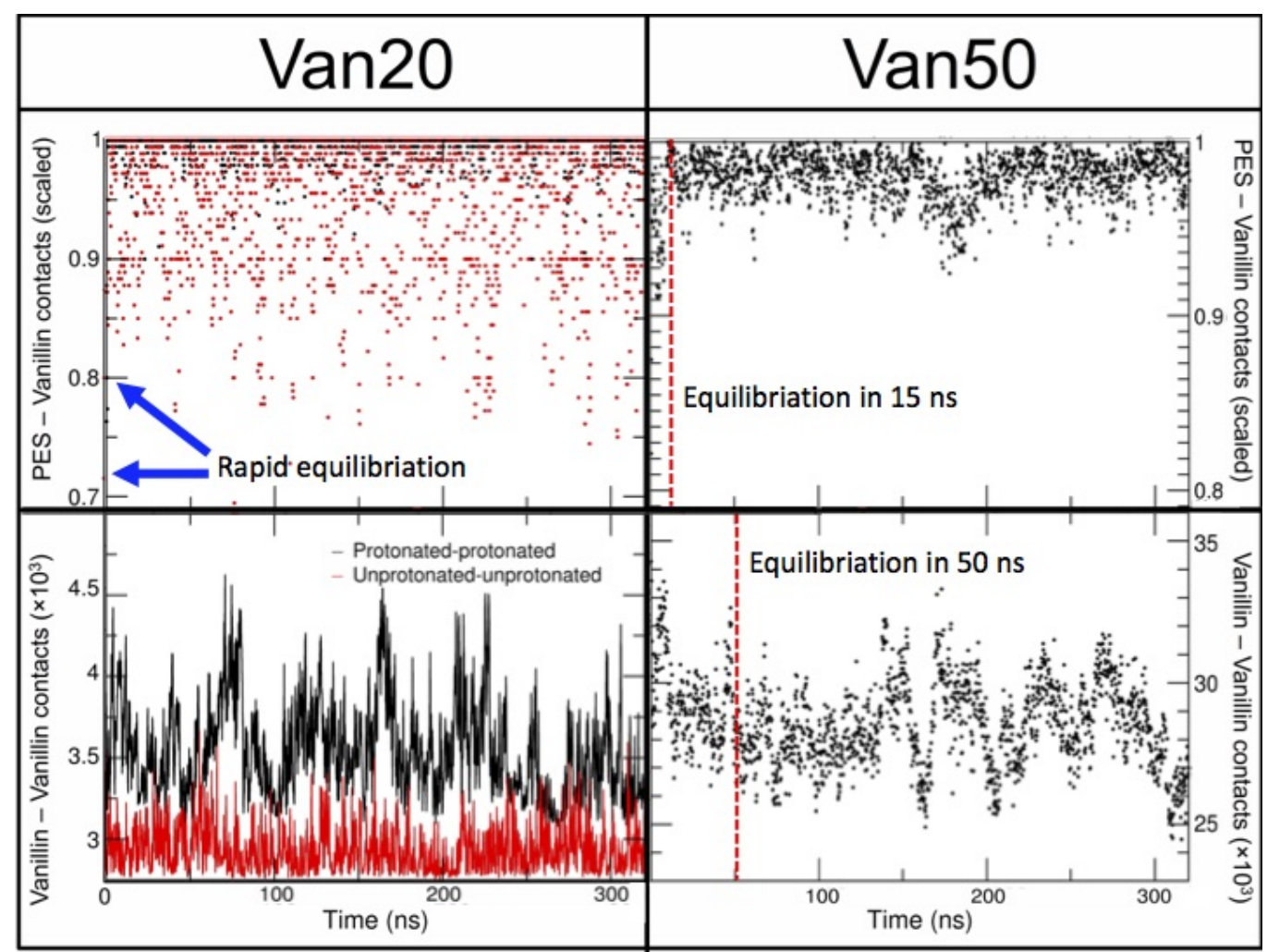

Figure S3: Results for PES-vanillin and vanillin-vanillin contacts as a function of time for both the Van20 and Van50 systems. For the Van20 systems, contacts involving protonated vanillin are shown in black, while the unprotonated vanillin contacts are shown in red. Note, 1) the near complete contact between protonated vanillin and PES and 2) that the number contacts between protonated vanillin molecules is higher than that between unprotonated vanillin molecules. 